\title{
A CONSISTENCY RESULT ON THIN-TALL SUPERATOMIC BOOLEAN ALGEBRAS
}

\author{
JUAN CARLOS MARTÍNEZ
}

(Communicated by Andreas R. Blass)

\begin{abstract}
We prove that if $\varkappa$ is an infinite cardinal with $\varkappa^{<\varkappa}=\varkappa$, then there is a cardinal-preserving notion of forcing that forces the existence of a $\varkappa$-thin-tall superatomic Boolean algebra. Consistency for specific $\varkappa$, like $\omega_{1}$, then follows as a corollary.
\end{abstract}

A superatomic Boolean algebra (abbreviated $\mathrm{sBa}$ ) is a Boolean algebra in which every subalgebra is atomic. It is known that a Boolean algebra $B$ is superatomic iff its Stone space $S(B)$ is scattered. The Cantor-Bendixson process for topological spaces can be transferred to the context of Boolean algebras, obtaining in this way a sequence of ideals, which are called the Cantor-Bendixson ideals. Suppose that $B$ is a Boolean algebra. Then, for every ordinal $\alpha$, we define by transfinite induction, the ideal $I_{\alpha}$ as follows. We put $I_{0}=\{0\}$. If $\alpha=\beta+1$, let $I_{\alpha}$ be the ideal generated by $I_{\beta}$ together with all $b \in B$ such that $b / I_{\beta}$ is an atom in $B / I_{\beta}$. If $\alpha$ is limit, $I_{\alpha}=\bigcup\left\{I_{\beta}: \beta<\alpha\right\}$. Then, $B$ is an $\mathrm{sBa}$ iff $B=I_{\alpha}$ for some $\alpha$.

The height of an $\mathrm{sBa} B, \operatorname{ht}(B)$, is the least ordinal $\alpha$ such that $B / I_{\alpha}$ is finite (which means $B=I_{\alpha+1}$ ). For every $\alpha<\operatorname{ht}(B)$ let $\operatorname{wd}_{\alpha}(B)$ be the cardinality of the set of atoms in $B / I_{\alpha}$. The width of $B, \operatorname{wd}(B)$, is the supremum of the $\operatorname{wd}_{\alpha}(B)$ for $\alpha<\operatorname{ht}(B)$. Then, for every infinite cardinal $\varkappa, B$ is called $\varkappa$-thin-tall, if $\operatorname{ht}(B)=\varkappa^{+}$and $\operatorname{wd}(B)=\varkappa$.

The reader may find in [4] a wide list of results on superatomic Boolean algebras, as well as a discussion of equivalent definitions and basic facts. In particular, it is known that it is possible to construct an $\omega$-thin-tall sBa with no extra set-theoretic axioms. This was proved by Rajagopalan and, independently, by Juhász and Weiss. On the other hand, Baumgartner and Shelah proved in [1] that it is consistent with the axioms of set theory that there exists an $\mathrm{sBa} B$ such that $\operatorname{ht}(B)=\omega_{2}$ and $\operatorname{wd}(B)=\omega$. The argument employed by Baumgartner and Shelah uses the fact that the forcing conditions are finite. However, if we want to prove, for an uncountable cardinal $\varkappa$, that the existence of a $\varkappa$-thin-tall $\mathrm{sBa}$ is consistent with the axioms of set theory, then we have to consider infinite forcing conditions. In this paper we see a modification of the argument given in [1], which permits us to deal with infinite forcing conditions. The set-theoretic

Received by the editors February 12, 1990 and, in revised form, November 14, 1990.

1980 Mathematics Subject Classification (1985 Revision). Primary 03E35, 06E99. 
terminology used here is taken from [1]. Our aim is to prove the following result.

Theorem. If $\varkappa$ is an infinite cardinal with $\varkappa^{<\varkappa}=\varkappa$, then there is a cardinalpreserving notion of forcing that forces the existence of a $\varkappa$-thin-tall $s B a$.

Proof. Let $\varkappa$ be an infinite cardinal with $\varkappa^{<\varkappa}=\varkappa$. Note that this implies, by König's lemma, that $\varkappa$ is regular. We define a partial ordering $P_{\varkappa}$, which does not depend on any special function, and we prove that forcing with $P_{\varkappa}$ preserves cardinals and adjoins a $\varkappa$-thin-tall sBa.

We put $T=\varkappa^{+} \times \varkappa$ and, for every $\alpha<\varkappa^{+}, T_{\alpha}=\{\alpha\} \times \varkappa . P_{\varkappa}$ adjoins a partial ordering $\leq$ on $T$ and a function $i$ on the set $\{\{s, t\}: s, t \in T\}$ such that the supremum of $i\{s, t\}$ represents the meet $s \wedge t$. We define $P_{\varkappa}$ as the set of all $p=\left(x_{p}, \leq_{p}, i_{p}\right)$ satisfying the following conditions:

(1) $x_{p}$ is a subset of $T$ of cardinality $<\varkappa$.

(2) $\leq_{p}$ is a partial ordering of $x_{p}$ such that if $s \in T_{\alpha}, t \in T_{\beta}$, and $s<_{p} t$, then $\alpha<\beta$.

(3) $i_{p}:\left\{\{s, t\}: s, t \in x_{p}\right\} \rightarrow\left\{x: x\right.$ is a finite subset of $\left.x_{p}\right\}$ satisfies the following:

(3.1) If $s \in T_{\alpha}, t \in T_{\beta}$, and $\alpha \leq \beta$ then:

(3.1.1) If $s=t$, then $i_{p}\{s, t\}=\{s\}$.

(3.1.2) If $s \neq t$ and $\alpha=\beta$, then $i_{p}\{s, t\}=0$.

(3.1.3) If $s<_{p} t$, then $i_{p}\{s, t\}=\{s\}$

(3.1.4) If $\alpha<\beta$ and $s \Varangle_{p} t$, then $i_{p}\{s, t\} \subseteq x_{p} \cap \bigcup\left\{T_{\tau}: \tau<\alpha\right\}$.

(3.2) For every $s, t \in x_{p}$ the following hold:

(3.2.1) If $u \in i_{p}\{s, t\}$, then $u \leq_{p} s, t$.

(3.2.2) If $v \leq_{p} s, t$, then there is a $u \in i_{p}\{s, t\}$ with $v \leq_{p} u$. $i_{q}$.

Now we put $p \leq_{\varkappa} q$ iff $x_{p} \supseteq x_{q}, \leq_{p}\left\lceil x_{q}=\leq_{q}\right.$ and $i_{p} \uparrow\left\{\{s, t\}: s, t \in x_{q}\right\}=$

Then proceeding in a way similar to that for [1, Theorem 7.1], one can prove that if $P_{\varkappa}$ preserves cardinals, then $P_{\varkappa}$ adjoints a $\varkappa$-thin-tall sBa.

Our aim is to show that forcing with $P_{\varkappa}$ preserves cardinals. Note that $P_{\varkappa}$ is $\varkappa$-closed. Then, our purpose is to prove that $P_{\varkappa}$ satisfies the $\varkappa^{+}$-chain condition. Suppose on the contrary that there exists an antichain $A$ of cardinality $\varkappa^{+}$. For every $p \in A$, we put $\gamma_{p}=\left\{\alpha: x_{p} \cap T_{\alpha} \neq 0\right\}$. Then, by the $\Delta$-system lemma (see [3, Theorem II.1.6]), we may assume that the $\gamma_{p}$ form a $\Delta$-system with kernel $\Delta$. Since the cardinality of every $\gamma_{p}$ is $<\varkappa$ and, for all $\alpha, \beta \in \Delta$ with $\alpha<\beta$, the cardinality of $\beta-\alpha$ is $\leq \varkappa$, we may also assume that $\Delta$ is an initial segment of $\gamma_{p}$ for every $p \in A$. Then by thinning out $A$ again if necessary, we may suppose that there is an ordinal $\gamma^{(1)}<\varkappa$ such that the order type of $\gamma_{p}-\Delta$ is $\gamma^{(1)}$ for every $p \in A$. Now we define $\gamma^{(0)}=$ supremum $\{\alpha+1: \alpha \in \Delta\}$, and $\gamma=\left(\gamma^{(0)}+\gamma^{(1)}\right)-\gamma^{(0)}$. Note that, since the cardinality of $\gamma$ is $<\varkappa$, we may assume that $\gamma_{p} \cap \gamma=0$ for every $p \in A$. Now, for every $p, q \in A$, we consider the unique order-preserving bijection $\pi_{p q}: \gamma_{p} \rightarrow \gamma_{q}$. Then, since the cardinality of each $x_{p}$ is $<\varkappa$, we may suppose that $\pi_{p q}$ lifts to an isomorphism of $x_{p}$ with $x_{q}$ given by $\pi_{p q}(\alpha, \beta)=\left(\pi_{p q}(\alpha), \beta\right)$. Finally we may also assume that, for every $p, q \in A$ and $s, t \in x_{p}$, we have:

$$
s \leq_{p} t \quad \text { iff } \pi_{p q}(s) \leq_{q} \pi_{p q}(t)
$$


and

$$
i_{q}\left(\pi_{p q}(s), \pi_{p q}(t)\right)=\left\{\pi_{p q}(u): u \in i_{p}\{s, t\}\right\} .
$$

Now we prove that the elements of $A$ are all compatible. Let $p, q \in A$. We construct an $r \in P_{\varkappa}$ such that $r \leq_{x} p$ and $r \leq_{\varkappa} q$. Let $\rho: \gamma \rightarrow\left(\gamma_{p}-\Delta\right)$ and $\mu: \gamma \rightarrow\left(\gamma_{q}-\Delta\right)$ the corresponding order-preserving bijections. For each $\alpha \in \gamma$, we set

$$
x^{(\alpha)}=\left\{(\alpha, \beta) \in T_{\alpha}:(\rho(\alpha), \beta) \in x_{p}\right\}=\left\{(\alpha, \beta) \in T_{\alpha}:(\mu(\alpha), \beta) \in x_{q}\right\} .
$$

Then we put

$$
x_{r}=x_{p} \cup x_{q} \cup \bigcup\left\{x^{(\alpha)}: \alpha \in \gamma\right\} .
$$

Now we make the following definitions:

$$
\begin{aligned}
x & =\bigcup\left\{x_{p} \cap T_{\alpha}: \alpha \in \Delta\right\}=\bigcup\left\{x_{q} \cap T_{\alpha}: \alpha \in \Delta\right\}, \\
y & =\bigcup\left\{x^{(\alpha)}: \alpha \in \gamma\right\}, \\
z_{1} & =\bigcup\left\{x_{p} \cap T_{\alpha}: \alpha \in \gamma_{p}-\Delta\right\}, \\
z_{2} & =\bigcup\left\{x_{q} \cap T_{\alpha}: \alpha \in \gamma_{q}-\Delta\right\} .
\end{aligned}
$$

Note that $\rho$ and $\mu$ lift to the isomorphisms of $y$ with $z_{1}$ and $y$ with $z_{2}$, respectively, given by $\rho(\alpha, \beta)=(\rho(\alpha), \beta)$ and $\mu(\alpha, \beta)=(\mu(\alpha), \beta)$. Then we define $\leq_{r}$ as follows: $s \leq_{r} t$ iff $s \leq_{p} t$ or $s \leq_{q} t$ or one of the following conditions holds:

(a) $s \in x, t \in y$, and $s \leq p \rho(t)$;

(b) $s, t \in y$ and $\rho(s) \leq_{p} \rho(t)$;

(c) $s \in y, t \in z_{1}$, and $\rho(s) \leq_{p} t$;

(d) $s \in y, t \in z_{2}$, and $\mu(s) \leq_{q} t$.

We show that $\leq_{r}$ is a transitive order. Suppose that $s \leq_{r} t \leq_{r} u$. The cases $s, t, u \in x_{p}$ and $s, t, u \in x_{q}$ are obvious. If $s \in x, t \in y$, and $u \in z_{1}$, we have that $s \leq_{p} \rho(t) \leq_{p} u$, whence $s \leq_{r} u$. Suppose that $s, t \in y$ and $u \in z_{1}$. It follows that $\rho(s) \leq_{p} \rho(t) \leq_{p} u$, and hence $s \leq_{r} u$. Now assume that $s, t \in y$ and $u \in z_{2}$. Since $\rho(s) \leq_{p} \rho(t)$, we have $\pi_{p q}(\rho(s)) \leq_{q} \pi_{p q}(\rho(t))$. But note that $\pi_{p q}(\rho(s))=\mu(s)$ and $\pi_{p q}(\rho(t))=\mu(t)$. Therefore $\mu(s) \leq_{q} \mu(t) \leq_{q} u$, and so $s \leq_{r} u$. The other cases are proved in a similar way.

Now we define $i_{r}$. Let $s, t \in x_{r}$. If $s, t \in x_{p}$, we put $i_{r}\{s, t\}=i_{p}\{s, t\}$. If $s, t \in x_{q}$, then $i_{r}\{s, t\}=i_{q}\{s, t\}$. If $s \in x$ and $t \in y$, we set $i_{r}\{s, t\}=$ $i_{p}\{s, \rho(t)\}$. If $s, t \in y$, then $i_{r}\{s, t\}=\left(i_{p}\{\rho(s), \rho(t)\} \cap x\right) \cup\left\{\rho^{-1}(u): u \in\right.$ $\left.i_{p}\{\rho(s), \rho(t)\}-x\right\}$. If $s \in y$ and $t \in z_{1}$, then $i_{r}\{s, t\}=i_{r}\left\{s, \rho^{-1}(t)\right\}$. Analogously if $s \in y$ and $t \in z_{2}$, then $i_{r}\{s, t\}=i_{r}\left\{s, \mu^{-1}(t)\right\}$. Finally, if $s \in z_{1}$ and $t \in z_{2}$, we put $i_{r}\{s, t\}=i_{r}\left\{\rho^{-1}(s), \mu^{-1}(t)\right\}$.

Note that $i_{r}$ is well defined. For example, if $s \in z_{1}, t \in z_{2}$, and $\rho^{-1}(s)=$ $\mu^{-1}(t)$, then $i_{r}\{s, t\}=\left\{\rho^{-1}(s)\right\}$. On the other hand, it should be noted that if $s \in y$ and $t \in z_{1}$, then $i_{r}\{s, t\}=\left(i_{p}\{\rho(s), t\} \cap x\right) \cup\left\{\rho^{-1}(u): u \in i_{p}\{\rho(s), t\}-\right.$ $x\}$, and analogously, if $s \in y$ and $t \in z_{2}$, then $i_{r}\{s, t\}=\left(i_{q}\{\mu(s), t\} \cap x\right) \cup$ $\left\{\mu^{-1}(u): u \in i_{q}\{\mu(s), t\}-x\right\}$.

In order to show that $r=\left(x_{r}, \leq_{r}, i_{r}\right) \in P_{\varkappa}$, we must verify condition (3). The easy proof of (3.1) is left to the reader. We prove condition (3.2.1). Let $s, t \in x_{r}$ and $u \in i_{r}\{s, t\}$. The cases $s, t \in x_{p}$ and $s, t \in x_{q}$ are obvious. For the rest, we consider three cases.

Case 1. $s \in x$ and $t \in y$. Then $u \in i_{p}\{s, \rho(t)\}$, and therefore $u \leq_{p} s, \rho(t)$, whence $u \leq_{r} s, t$. 
Case 2. $s, t \in y$. If $u \in x$, then $u \in i_{p}\{\rho(s), \rho(t)\}$, and hence $u \leq_{p}$ $\rho(s), \rho(t)$, whence $u \leq_{r} s, t$.

If $u \in y$, then $\rho(u) \in i_{p}\{\rho(s), \rho(t)\}$, and so $\rho(u) \leq_{p} \rho(s), \rho(t)$, which implies $u \leq_{r} s, t$.

If $s \in y$ and $t \in z_{1}$, or $s \in y$ and $t \in z_{2}$, the considerations are similar to those of Case 2.

Case 3. $s \in z_{1}$ and $t \in z_{2}$. We have that $u \in i_{r}\left\{\rho^{-1}(s), \mu^{-1}(t)\right\}$ and then by Case $2, u \leq_{r} \rho^{-1}(s), \mu^{-1}(t)$. If $u \in x$, it is clear that $u \leq_{r} s, t$. If $u \in y$, we infer than $\rho(u) \leq_{p} s$ and $\mu(u) \leq_{q} t$, which implies $u \leq_{r} s, t$.

Now we check (3.2.2). Let us consider $s, t \in x_{r}$ and $v \leq_{r} s, t$. The case $s \in X$ and $t \in x_{p} \cup x_{q}$ is obvious. For the rest, we consider four cases.

Case 1. $s \in x$ and $t \in y$. It follows that $v \leq_{p} s, \rho(t)$, and thus there is a $u \in i_{p}\{s, \rho(t)\}=i_{r}\{s, t\}$ such that $v \leq_{r} u$.

Case 2. $s, t \in y$. First suppose that $v \in x$. Then $v \leq_{p} \rho(s), \rho(t)$, and therefore there is a $u \in i_{p}\{\rho(s), \rho(t)\}$ such that $v \leq_{p} u$. If $u \in x$, then $u \in i_{r}\{s, t\}$. And if $u \in z_{1}$, we infer that $v \leq_{r} \rho^{-1}(u)$ and $\rho^{-1}(u) \in i_{r}\{s, t\}$.

Now suppose that $v \in y$. Then $\rho(v) \leq_{p} \rho(s), \rho(t)$, and hence there is a $u \in i_{p}\{\rho(s), \rho(t)\}$ such that $\rho(v) \leq_{p} u$, whence $v \leq_{r} \rho^{-1}(u)$ and $\rho^{-1}(u) \in$ $i_{r}\{s, t\}$.

The cases $s \in y, t \in z_{1}$ and $s \in y, t \in z_{2}$ can be verified by means of an argument similar to the one given in Case 2 .

Case 3. $s, t \in z_{1}$. If $v \in x_{p}$ we are done. Then suppose that $v \in y$. It follows that $\rho(v) \leq_{p} s, t$, and hence there is a $u \in i_{p}\{s, t\}$ such that $\rho(v) \leq_{p}$ $u$. But $\rho(v) \leq_{p} u$ implies $v \leq_{r} u$.

The case $s, t \in z_{2}$ is similar to Case 3 .

Case 4. $s \in z_{1}$ and $t \in z_{2}$. It is easy to infer that $v \leq_{r} \rho^{-1}(s), \mu^{-1}(t)$, and then by Case 2, there is a $u \in i_{r}\left\{\rho^{-1}(s), \mu^{-1}(t)\right\}=i_{r}\{s, t\}$ such that $v \leq_{r} u$.

This completes the verification of $(3)$ and the proof that $P_{\varkappa}$ has the $\varkappa^{+}$-chain condition.

Remarks. (1) Juhász and Weiss proved in [2] that, for every ordinal $\alpha<\omega_{2}$, there exists an $\mathrm{sBa} B_{\alpha}$ such that $\operatorname{ht}\left(B_{\alpha}\right)=\alpha$ and $\operatorname{wd}\left(B_{\alpha}\right)=\omega$. Then, by using the well-known fact that there is an almost disjoint family of $2^{\omega}$ subsets of $\omega$, we obtain that $\rceil \mathrm{CH}$ implies the existence of an sBa with exactly $\omega$ atoms and height $\omega_{2}$. On the other hand, since the partial ordering $P_{\omega_{1}}$ is countably closed, we infer that forcing with $P_{\omega_{1}}$ preserves $\mathrm{CH}$ (see [3, Theorem VII.6.14]), and thus we obtain as a corollary that the existence of an $\omega_{1}$-thin-tall sBa is consistent with $\mathrm{ZFC}+\mathrm{CH}$.

(2) Suppose that $\varkappa, \lambda$ are infinite cardinals such that $\varkappa^{<\varkappa}=\varkappa$ and $\varkappa<\lambda$. Then, it is consistent with the axioms of set theory that there exists an $\mathrm{sBa} B$ such that $\operatorname{ht}(B)=\varkappa+1, \operatorname{wd}_{\alpha}(B)=\varkappa$ for every $\alpha<\varkappa$ and $\operatorname{wd}_{\varkappa}(B)=\lambda$. This result can be proved by means of an argument similar to the one given before. However, if we assume that the ground model satisfies $\mathrm{GCH}$, it is easier to show this fact, if we use the argument given in [5, Theorem 9]. More precisely, let us consider a cardinal-preserving generic extension $N$ such that, in $N, 2^{\varkappa} \geq \lambda$ and $2^{\varkappa_{0}}=\varkappa_{0}^{+}$for every cardinal $\varkappa_{0}<\varkappa$ (see [3, Theorem VII.6.17]). Then in $N$, the complete binary tree of height $\varkappa$ is a $\varkappa$-Canadian tree with at least $\lambda$ paths. But note that the existence of such a tree implies the existence of the required $\mathrm{sBa}$. 


\section{ACKNOWLEDGMENT}

I wish to express my gratitude to I. Juhász for bringing the problem considered here to my attention.

\section{REFERENCES}

1. J. Baumgartner and S. Shelah, Remarks on superatomic Boolean algebras, Ann. Pure Appl. Logic 33 (1987), 109-129.

2. I. Juhász and W. Weiss, On thin-tall scattered spaces, Colloq. Math. 90 (1978), 64-68.

3. K. Kunen, Set theory: an introduction to independence proofs, North-Holland, Amsterdam, 1980.

4. J. Roitman, Superatomic Boolean algebras, Handbook of Boolean Algebras (J. D. Monk and R. Bonnet, eds.), North-Holland, Amsterdam, 1989, pp. 719-740.

5. M. Weese, On cardinal sequences of Boolean algebras, Algebra Universalis 23 (1986), 85-97.

Facultad de Matemáticas, Universidad de Barcelona, 08007 Barcelona, Spain 